J3eA - Vol. 3 - 3 (2004).

DOI : 10.1051/bib-j3ea:2004003

\title{
Instrumentation virtuelle et moteur asynchrone en vitesse variable
}

\author{
J.J. Huselstein * et C. Glaize ** (Université de Montpellier II) \\ Mis en ligne le 15/03/2004.
}

\begin{abstract}
Résumé
Dans cet article, nous présentons les aspects pédagogiques et techniques d'un banc de mesure basé sur micro-ordinateur et utilisé en travaux pratiques de génie électrique. Cette instrumentation est utilisable pour de nombreuses applications. Nous nous intéresserons ici d'abord à un redresseur en pont mixte triphasé. Puis nous appliquerons ce dispositif de mesure à un entraînement à vitesse variable constitué par une machine asynchrone à cage alimentée par un variateur de vitesse industriel. Nous montrerons alors que ce type d'instrumentation peut apporter des fonctionnalités que les appareils de mesure traditionnels ne peuvent pas offrir.
\end{abstract}

Mots-clés : motivation des étudiants, instrumentation virtuelle, acquisition de données, LabVIEW, machine asynchrone, variation de vitesse.

(C) EDP Sciences, 2004.

\footnotetext{
Niveau de connaissances requis. Grandeurs complexes et puissances en régime sinusoïdal équilibré, notions de base sur la machine asynchrone en vitesse variable.

Niveau des étudiants. Licence EEA, licence IE, maîtrise EEA, élèves ingénieurs, IUP GEII, ...

$\mathrm{Bac}+2$ pour les étudiants de la spécialité : DUT Génie Électrique option Électrotechnique, BTS

Électrotechnique.
}

\begin{abstract}
* Jean-Jacques Huselstein ${ }^{1}$, ancien élève de l'ENS de Cachan, agrégé de Génie Électrique en 1988, est actuellement maître de conférences à l'Université Montpellier II. Il enseigne dans les domaines de l'électronique de puissance, l'électronique de commande et l'électrotechnique en licence, en maîtrise et en DESS. Après avoir travaillé sur les convertisseurs matriciels, ses thèmes de recherche actuels au Laboratoire d'Électrotechnique de Montpellier portent sur la fiabilité des cellules de commutation de puissance à IGBT ainsi que sur les chaînes de conversion éoliennes.
\end{abstract}

e-mail : huselstein@univ-montp2.fr

a ** Christian Glaize ${ }^{1}$, ancien élève de l'ENS de Cachan, est professeur à l'Université Montpellier II et enseigne l'électrotechnique et l'électronique de puissance à l'IUT de Nîmes.

En recherche, il a créé et dirigé pendant quinze ans le Laboratoire d'Électrotechnique de Montpellier. Il y a travaillé et y travaille toujours sur l'amélioration de l'environnement avec et par l'électricité (traction électrique, véhicules électriques, énergies renouvelables, optimisation de l'éclairage urbain... ) et sur le stockage de l'énergie électrique, en particulier dans le domaine de la gestion de la charge et de la décharge.

Il est animateur de la Commission Enseignement du Club EEA et responsable de la médiathèque e-EEA.

e-mail : glaize@univ-montp2.fr (auteur de correspondance)

\footnotetext{
${ }^{1}$ Laboratoire d'Électrotechnique de Montpellier (CC 079), Université Montpellier II (Sciences et Techniques du Languedoc), Place Eugène Bataillon, F-34095 Montpellier CEDEX 5, France.
} 


\section{Introduction}

Pour attirer les étudiants, les motiver et les garder dans nos filières, il est devenu indispensable de moderniser nos méthodes d'enseignement. Ceci est tout particulièrement vrai pour les travaux pratiques. Dans l'industrie, les appareils de mesures classiques ont été remplacés par des systèmes de supervision basés sur l'outil informatique. Pourquoi nous contenterions-nous alors dans nos formations de techniques de mesures anciennes?

Ainsi, depuis plusieurs années, nos étudiants étudient en travaux pratiques le fonctionnement d'un véhicule électrique à l'aide d'un tel système de mesure embarqué $[1,2]$.

Dans cet article nous présentons un banc de mesure basé sur PC avec une application particulière à l'étude d'un entraînement en vitesse variable par un moteur asynchrone.

\section{Avantages de l'instrumentation virtuelle}

Des points de vue technique et économique, il est possible de réaliser, avec des instruments virtuels, davantage de mesures avec plus de précision et un coût moindre qu'avec des appareils classiques. L'outil de base est un micro-ordinateur, souvent déjà présent pour d'autres utilisations, auquel il faut ajouter une carte d'acquisition analogique (ici 8 entrées multiplexées, 1 CAN 12 bits $100 \mathrm{kéch} / \mathrm{s}$ ) et une interface de mesure comportant les capteurs de courant à effet Hall et les sondes de tension différentielles (conçues et réalisées en interne).

Les instruments virtuels peuvent remplacer de nombreux voltmètres, ampèremètres, wattmètres et commutateurs ainsi qu'un oscilloscope numérique multi-voies. Des fonctions de mesures plus avancées, inexistantes sur les appareils traditionnels, peuvent être programmées.

Les avantages pédagogiques sont très importants. En premier lieu, l'instrumentation sur ordinateur donne immédiatement aux étudiants une vision plus moderne et plus attractive de l'électrotechnique. Les mesures sont moins fastidieuses et beaucoup plus rapides. Les étudiants peuvent consacrer plus de temps à la compréhension des dispositifs et systèmes qu'ils étudient, les grandeurs que l'on avait l'habitude de déterminer par des méthodes détournées peuvent être présentées directement et visuellement.

\section{Exemples}

\subsection{Organisation générale}

La chaîne de mesure équipant chaque poste de travail est constituée autour d'une carte d'acquisition, logée dans le PC, comportant 8 entrées analogiques multiplexées. La liaison entre le système de traitement de l'énergie électrique à étudier et la carte d'acquisition se fait par l'intermédiaire d'une interface de mesure. La gestion de la carte d'acquisition, le traitement et l'affichage des mesures sont assurés par une application développée dans l'environnement de programmation graphique LabVIEW $[3,4]$.

\subsection{Interface de mesure universelle}

L'interface de mesure universelle (Fig. 1) a été réalisée par nos soins. Pour s'adapter à de nombreuses configurations rencontrées en électronique de puissance, elle comporte, dans un même boîtier, trois sondes de tensions différentielles et trois sondes de courant à effet Hall. Pour permettre des mesures d'autres grandeurs physiques (couple et vitesse par exemple), elle comporte également deux entrées directes (non isolées, plage d'entrée de $\pm 5 \mathrm{~V}$, bornes BNC). 


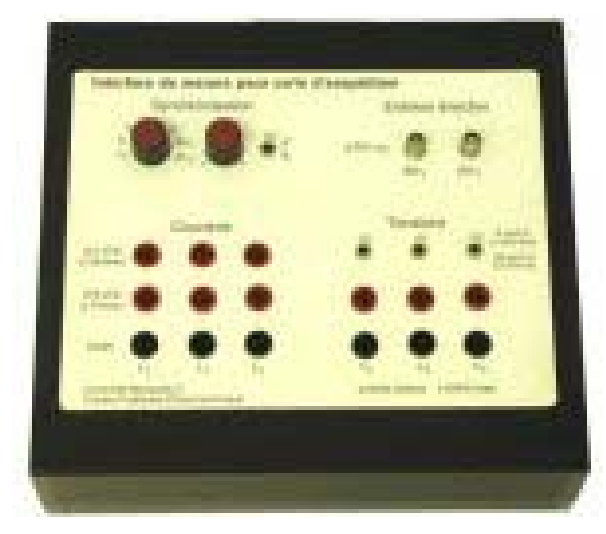

Fig. 1. Interface de mesure universelle.

Toutes les grandeurs analogiques sont transmises à la carte d'acquisition pour un fonctionnement dans une plage de tension d'entrée fixe et unique de $\pm 5 \mathrm{~V}$. Ceci permet le multiplexage des entrées sur une carte d'acquisition économique et une minimisation des effets de perturbations électromagnétiques.

Pour utiliser l'ensemble de mesure comme un oscilloscope, un dispositif générant le signal de synchronisation logique de la carte d'acquisition a été intégré dans l'interface de mesure. Il est construit autour d'un comparateur à hystérésis et s'utilise comme une synchronisation d'oscilloscope.

Lorsqu'il est nécessaire d'éliminer les harmoniques de découpage dues à la modulation de largeur d'impulsions du variateur, un boîtier comportant pour chaque voie un filtre passe-bas de Butterworth du deuxième ordre avec une fréquence de coupure de $500 \mathrm{~Hz}$ est inséré entre l'interface de mesure et la carte d'acquisition. Les 8 filtres étant identiques, les retards introduits sur chacune des grandeurs mesurées se compensent.

\section{Applications générales}

L'interface de mesures présentée peut être employée pour de nombreuses d'applications. Pour expliciter son fonctionnement, nous allons d'abord la présenter sur une structure simple. Elle est utilisée par nos étudiants dans le cadre de l'étude des redresseurs triphasés. La possibilité d'affichage simultanée en mode oscilloscope de 4 tensions et de 4 courants permet de comprendre plus facilement le fonctionnement des différentes structures de redresseurs. De plus, les appareils de mesures classiques sont devenus inutiles. Il suffit de sélectionner pour les voltmètres ou ampèremètres virtuels la voie et la fonction choisies (valeur moyenne ou valeur efficace). Les calculs de puissances moyennes (fonction wattmètre) se font par sélection de la voie tension et de la voie courant adaptées.

La figure 2 représente l'écran de mesures obtenu sur un redresseur triphasé en pont mixte. Dans la colonne de gauche apparaissent (de haut en bas), sous le titre « Voltmètres » :

- les valeurs efficaces de deux des trois tensions simples d'alimentation ;

- la valeur moyenne de la tension de sortie.

Sous le titre « Ampèremètres », on lit :

- les intensités efficaces de deux des trois courants de ligne ;

- la valeur moyenne du courant de sortie.

On lit ensuite deux puissances absorbées en ligne et la puissance de sortie (en continu). Ces puissances sont calculées comme la valeur moyenne du produit des valeurs instantanées $i_{i}$ et $v_{i}$.

Une des deux entrées directes a été utilisée avec une sonde de tension différentielle (1/100) extérieure pour visualiser la troisième tension simple (en jaune).

Au centre sont représentées les valeurs instantanées des tensions et courants mesurés. 
À droite, se trouvent des commandes (calibres, facteurs d'échelle des entrées directes, synchronisation de «l'oscilloscope », échelles des courbes) et légendes (couleurs des courbes en fonction des entrées). Les calibres des entrées doivent être sélectionnés par les étudiants.

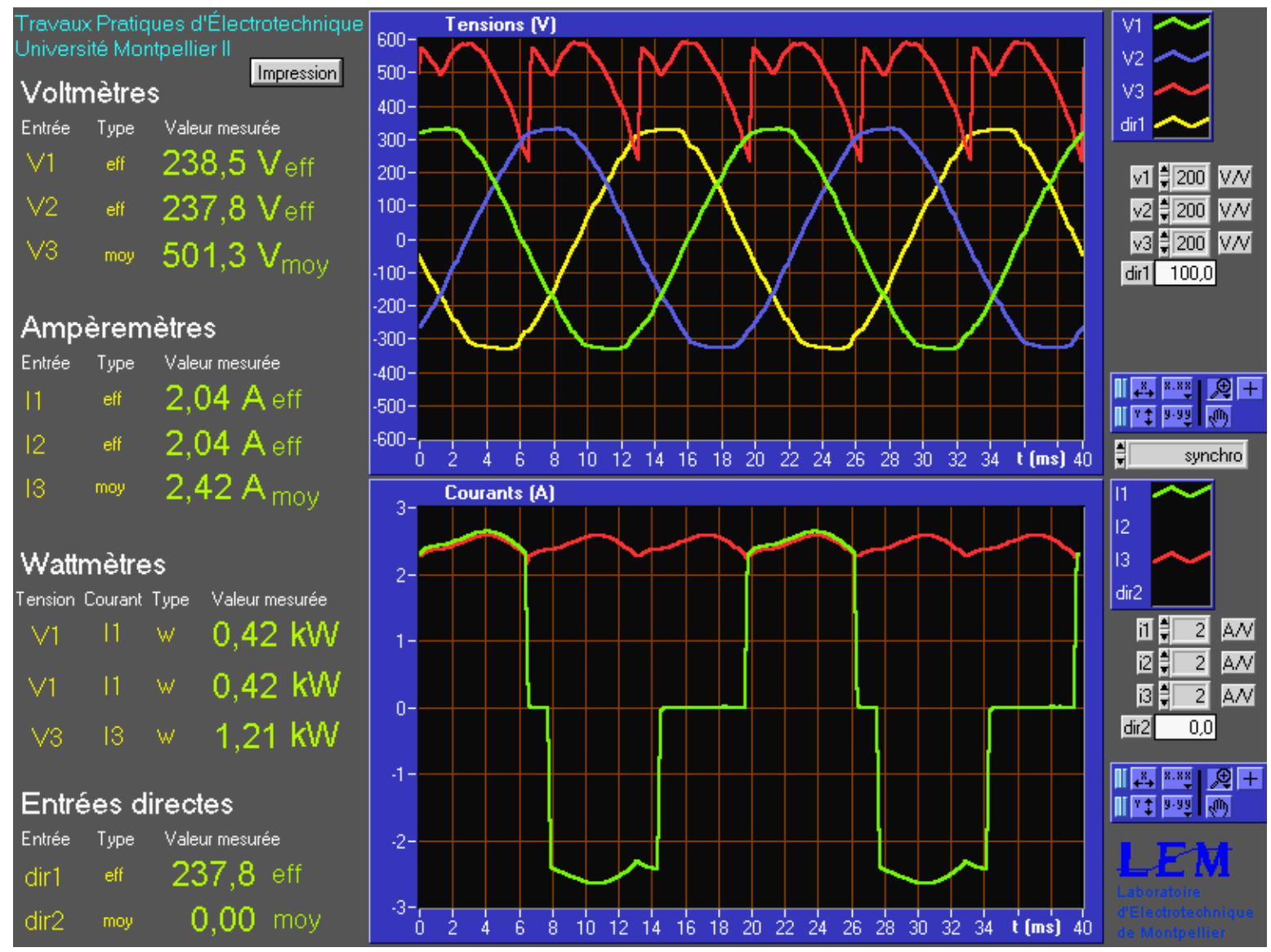

Fig. 2. Mesures sur un redresseurtriphasé en pont mixte.

\section{5. Étude d'un moteur asynchrone en vitesse variable}

\subsection{Banc moteur}

Le banc d'essai est constitué par un moteur asynchrone à cage alimenté à partir d'un variateur industriel et d'une charge mécanique formée par un volant d'inertie et un frein à poudre (Fig. 3).

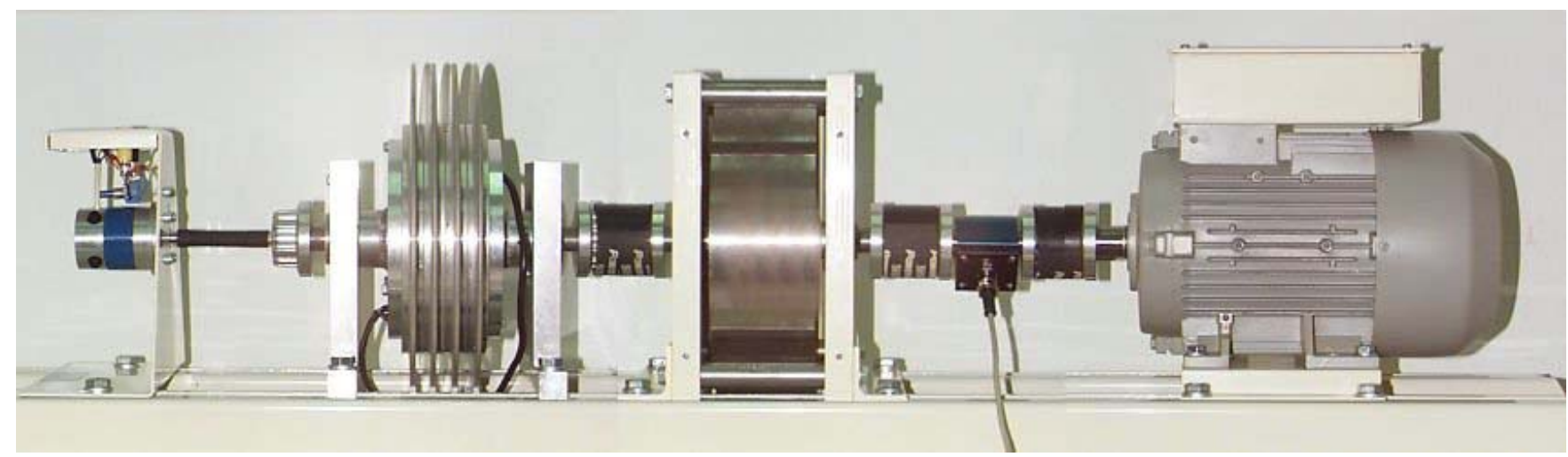

Fig. 3. Banc d'essai. 
Le but est de reproduire le comportement, statique et dynamique, d'une charge mécanique réelle (convoyeur à bande, tramway, etc.). Le volant d'inertie permet d'étudier des phénomènes transitoires longs, tels les phases d'accélérations et de décélérations (ces dernières permettent d'étudier le fonctionnement en génératrice du moteur asynchrone).

\subsection{Mesure du couple et de la vitesse}

Pour permettre les mesures des grandeurs mécaniques, couple et vitesse de rotation, le banc a été équipé d'un capteur de couple rotatif et d'une dynamo tachymétrique. Ces instruments sont reliés à un boîtier comportant les conditionneurs nécessaires et fournissent des signaux de sortie adaptés à la plage de tensions d'entrée de l'interface de mesure $( \pm 5 \mathrm{~V})$. Ce boîtier comporte également deux afficheurs pour une éventuelle utilisation autonome.

\subsection{Instruments virtuels classiques}

Il est possible d'étudier une machine asynchrone avec des instruments de mesures virtuels classiques, voltmètres, ampèremètres et wattmètres. Ceci permet d'enseigner ces méthodes de mesure (méthodes des 2 wattmètres... ) avec un câblage simplifié et une utilisation plus confortable et plus rapide.

\subsection{Mesures vectorielles}

Nous avons développé un autre instrument virtuel avec la même interface universelle et la même carte d'acquisition. Il s'agit d'un dispositif de mesure vectoriel permettant une approche rapide, précise et complète du fonctionnement en vitesse variable d'un moteur asynchrone dans tous les modes de fonctionnement. La « programmation » en LabVIEW est de type graphique. Le diagramme de cette application est donné en annexe.

Les étudiants câblent le moteur asynchrone au variateur en passant par les capteurs de courant de l'interface de mesure et en connectant ses entrées de tension différentielles. Les étudiants configurent ensuite les sensibilités de mesures. S'ils le souhaitent, ils peuvent modifier la fréquence d'échantillonnage et le nombre d'échantillons.

Les trois tensions composées $\mathrm{u}_{12}, \mathrm{u}_{23}$ et $\mathrm{u}_{31}$, les 3 tensions simples $\mathrm{v}_{1}$, $\mathrm{v}_{2}$ et $\mathrm{v}_{3}$ (obtenues par calcul) ainsi que les 3 courants $i_{1}, i_{2}$ et $i_{3}$ sont affichés comme sur un oscilloscope (Fig. 4a, 4b, 4c, 4d, 4e).

Un calcul vectoriel basé sur les phaseurs représentant le système de tensions et le système de courant permet d'obtenir toutes les grandeurs électriques du circuit monophasé équivalent. Les vecteurs courant et tension du circuit monophasé équivalent sont affichés dans le plan complexe (le vecteur tension étant pris comme origine des phases).

Les valeurs efficaces de la tension $\mathrm{V}$ et du courant I, leur déphasage $\phi$, les puissances active $\mathrm{P}$, réactive $\mathrm{Q}$, apparente $\mathrm{S}$ ainsi que la fréquence statorique (avec le signe représentant le sens de rotation des phases) sont indiquées par des afficheurs numérique et analogiques.

Les grandeurs mécaniques, vitesse de rotation et couple, sont également affichées sur le même écran.

Cet ensemble forme un « tableau de bord » fournissant clairement et en temps réel toutes les grandeurs électriques et mécaniques relatives au fonctionnement du moteur. Lors de régimes transitoires, accélération, décélération, tous les affichages de l'écran peuvent être figés par l'appui d'une touche pour permettre une analyse détaillée.

Les photos $4 \mathrm{a}$ et $4 \mathrm{~b}$ montrent le fonctionnement à vitesse nominale à vide et en charge. On « voit » simplement des propriétés de la machine asynchrone. À vide, le courant est (presque) en quadrature arrière par rapport à la tension. En charge, la partie réactive du courant reste constante et s'y ajoute une composante en phase avec la tension. On observe aussi que la tension d'alimentation en charge chute légèrement, ce qui fait chuter la composante réactive du courant et la puissance réactive. Bien entendu, la puissance active a fortement augmenté. 


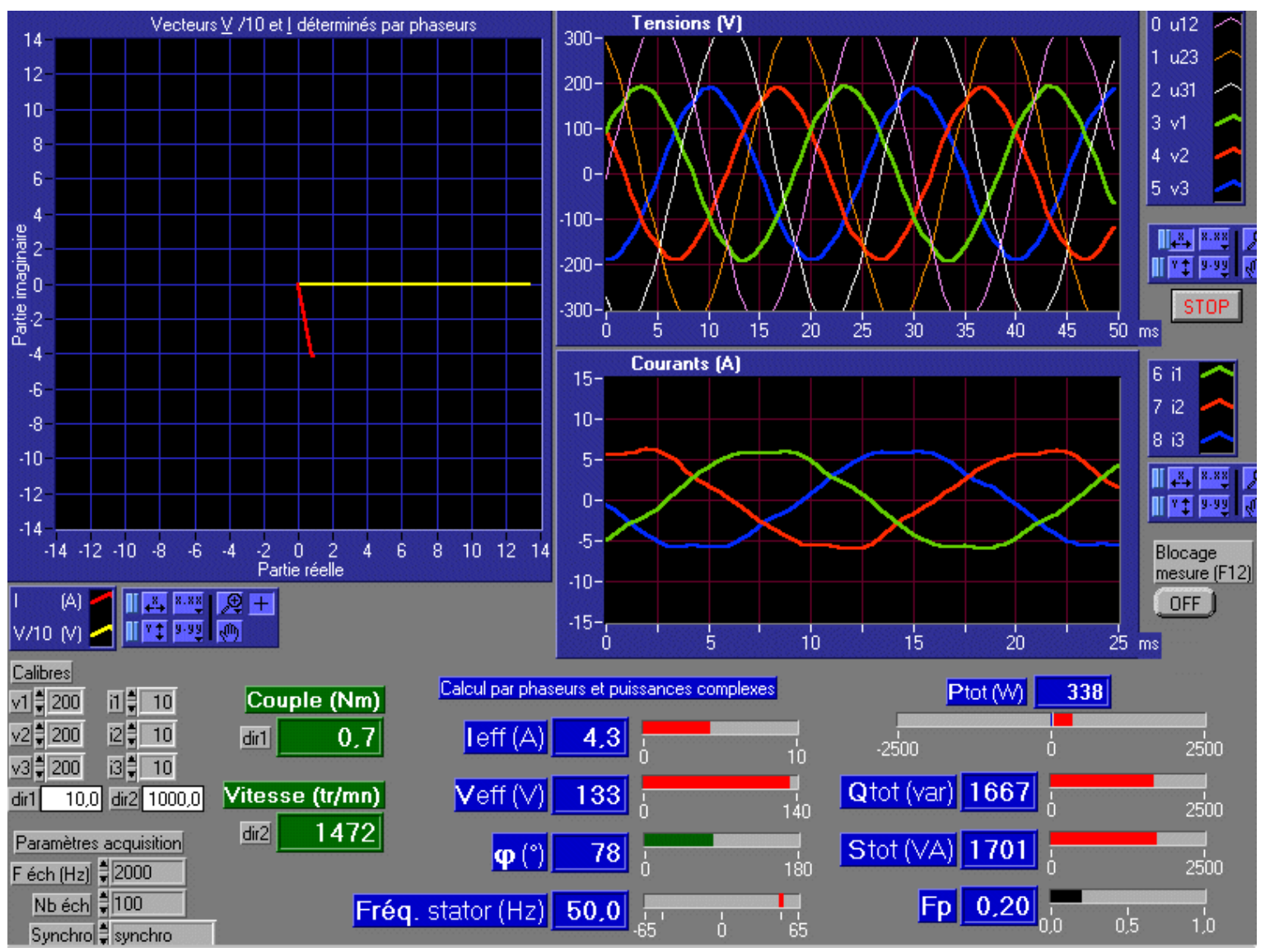

Fig. 4.a) Machine à vitesse nominale et à vide. 


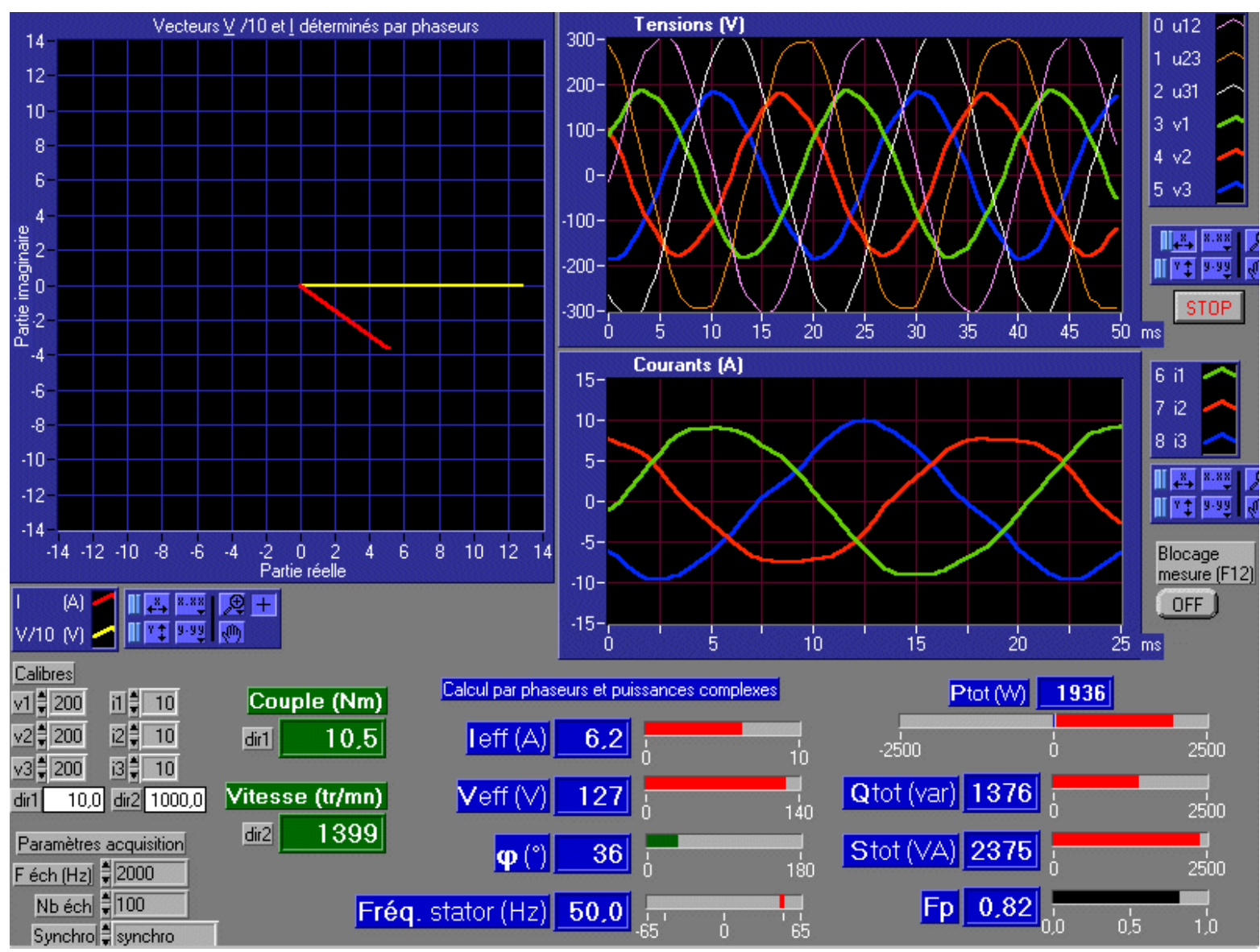

Fig. 4.b) Machine à vitesse nominale et en charge.

Le variateur utilisé permet de dépasser les $1500 \mathrm{tr} / \mathrm{min}$ (vitesse de synchronisme de la machine à $50 \mathrm{~Hz}$ ). C'est ce qu'illustrent les figures $4 \mathrm{c}$ (à vide) et $4 \mathrm{~d}$ (en charge). Pour un couple de sortie identique (10,5 Nm), la puissance active absorbée à $1862 \mathrm{tr} / \mathrm{min}$ est bien supérieure à celle absorbée à $1400 \mathrm{tr} / \mathrm{min}$. La proportionnalité n'est cependant pas tout à fait respectée car la commande en U/f du variateur n'est plus respectée au-delà de $50 \mathrm{~Hz}$ car la tension redressée est limitée par le redresseur d'entrée. 


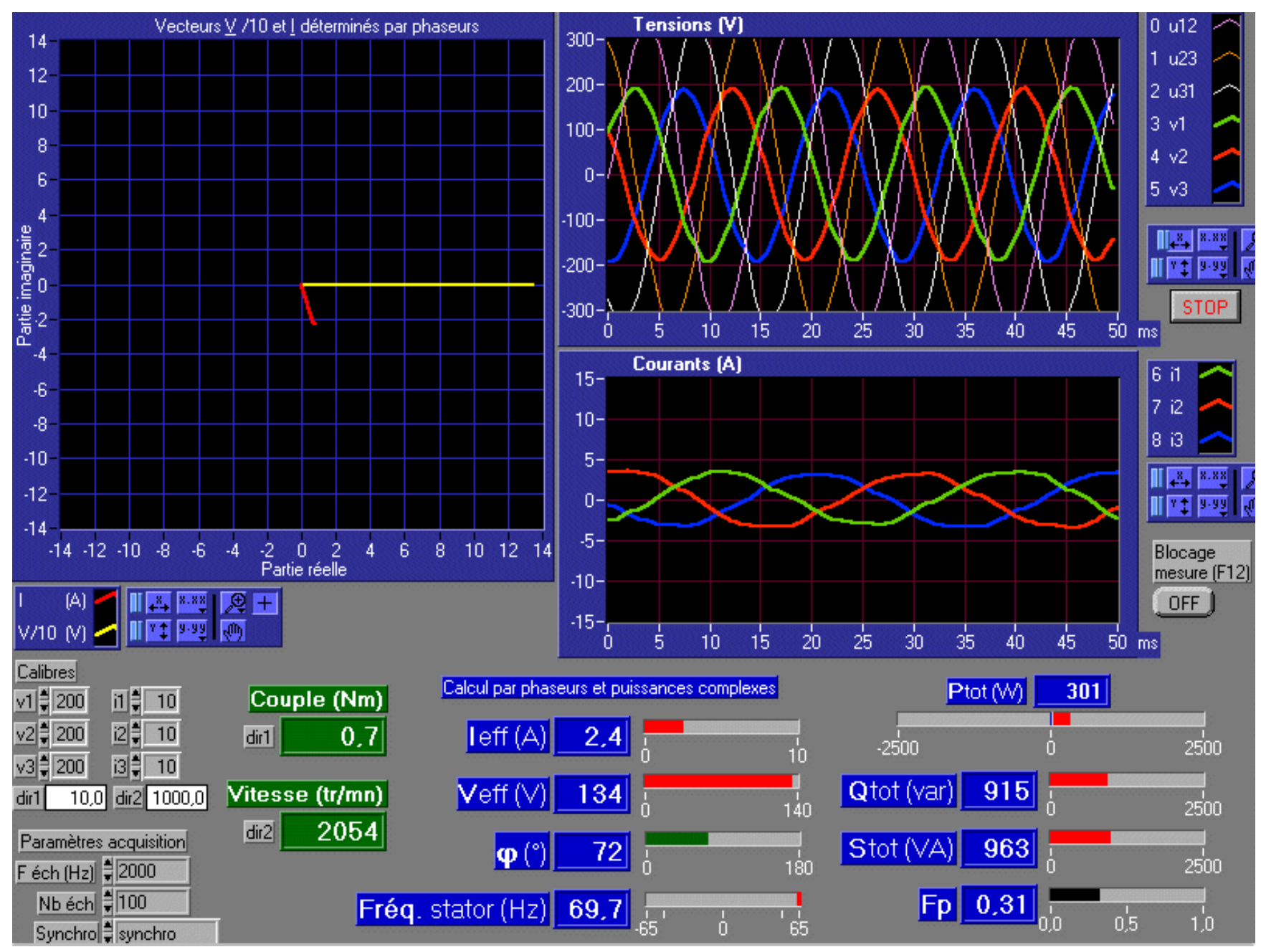

Fig. 4.c) Machine en survitesse et à vide. 


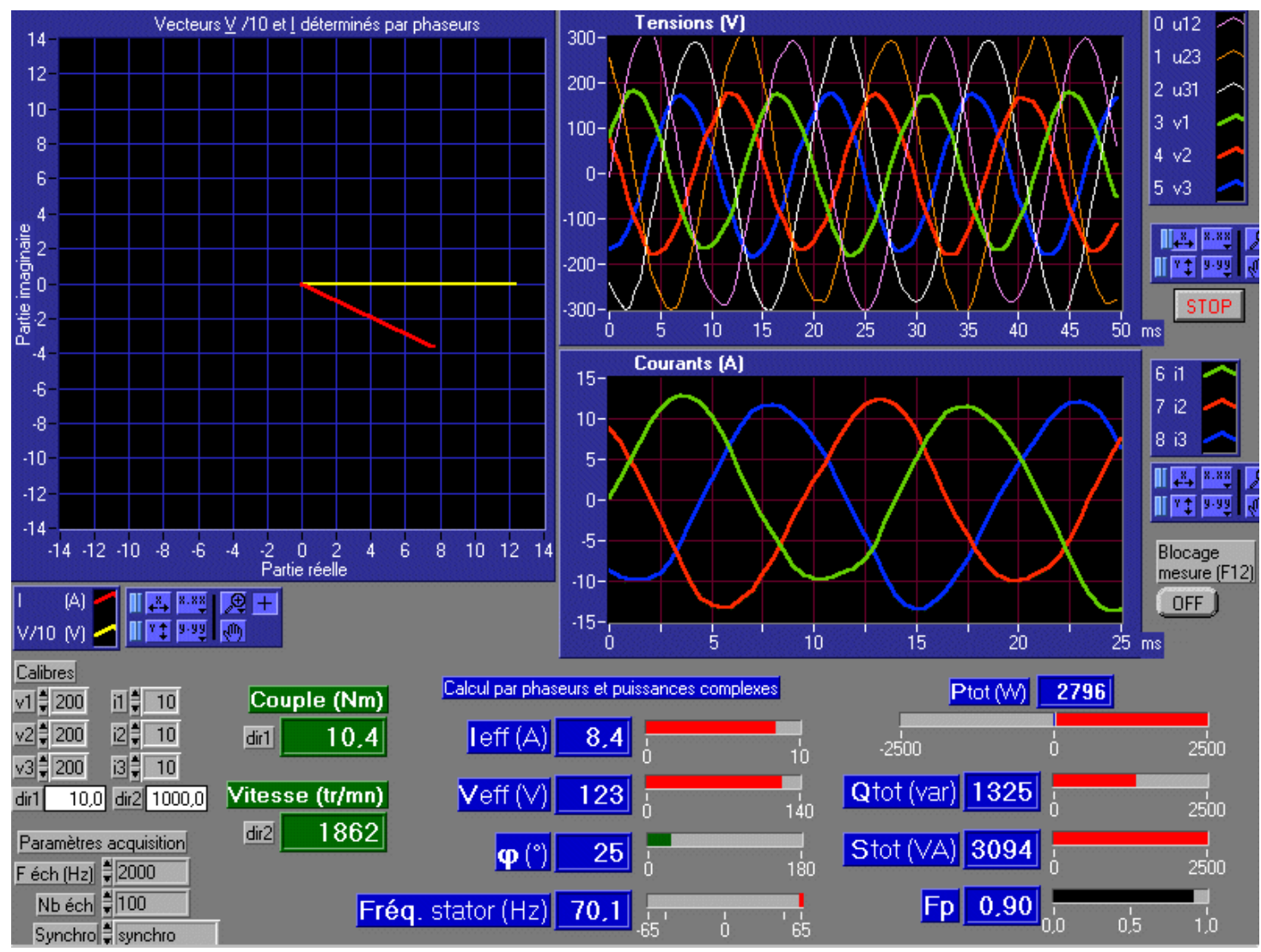

Fig. 4.d) Machine en survitesse et en charge.

La figure 4e montre un fonctionnement en freinage. Il est important de noter que ces mesures en génératrice sont faites en régime transitoire. C'est le volant d'inertie qui fournit l'énergie à la machine. En pouvant effectuer les calculs sur seulement quelques points de mesure, le programme permet d'éviter d'utiliser une charge réversible de type machine à courant continu alimentée. Cette possibilité de mesure en transitoire est un progrès important par rapport aux instruments de mesure traditionnels.

Dans cet essai, on voit que la partie active du courant change de signe. 


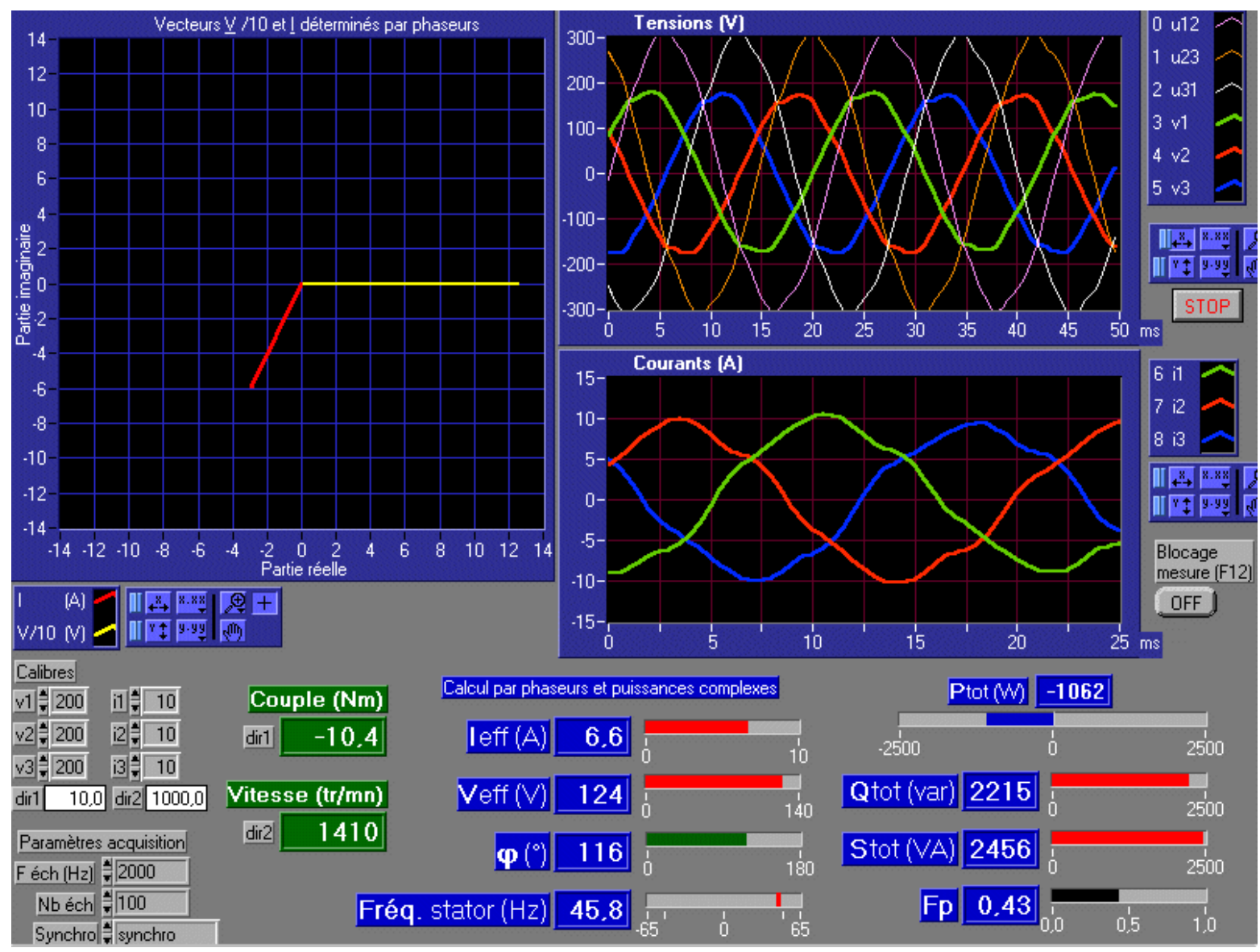

Fig. 4.e) Machine en freinage.

\subsection{Enregistrement de régimes transitoires}

Un autre instrument virtuel permet de visualiser aisément les fonctionnements transitoires. Sur l'enregistrement présenté (Fig. 5), la vitesse de rotation passe en 6 secondes de $+1500 \mathrm{tr} / \mathrm{min}$ à $-1500 \mathrm{tr} / \mathrm{min}$. De haut en bas, sont représentés :

- le courant instantané dans une phase d'alimentation du moteur ;

- la tension entre deux phases du moteur;

- le couple mécanique sur l'arbre du moteur ;

- la vitesse de rotation.

Note : on rappelle (voir Fig. 3) que la charge de la machine est ici le seul volant d'inertie, le frein à poudre n'est pas activé dans cet essai. 


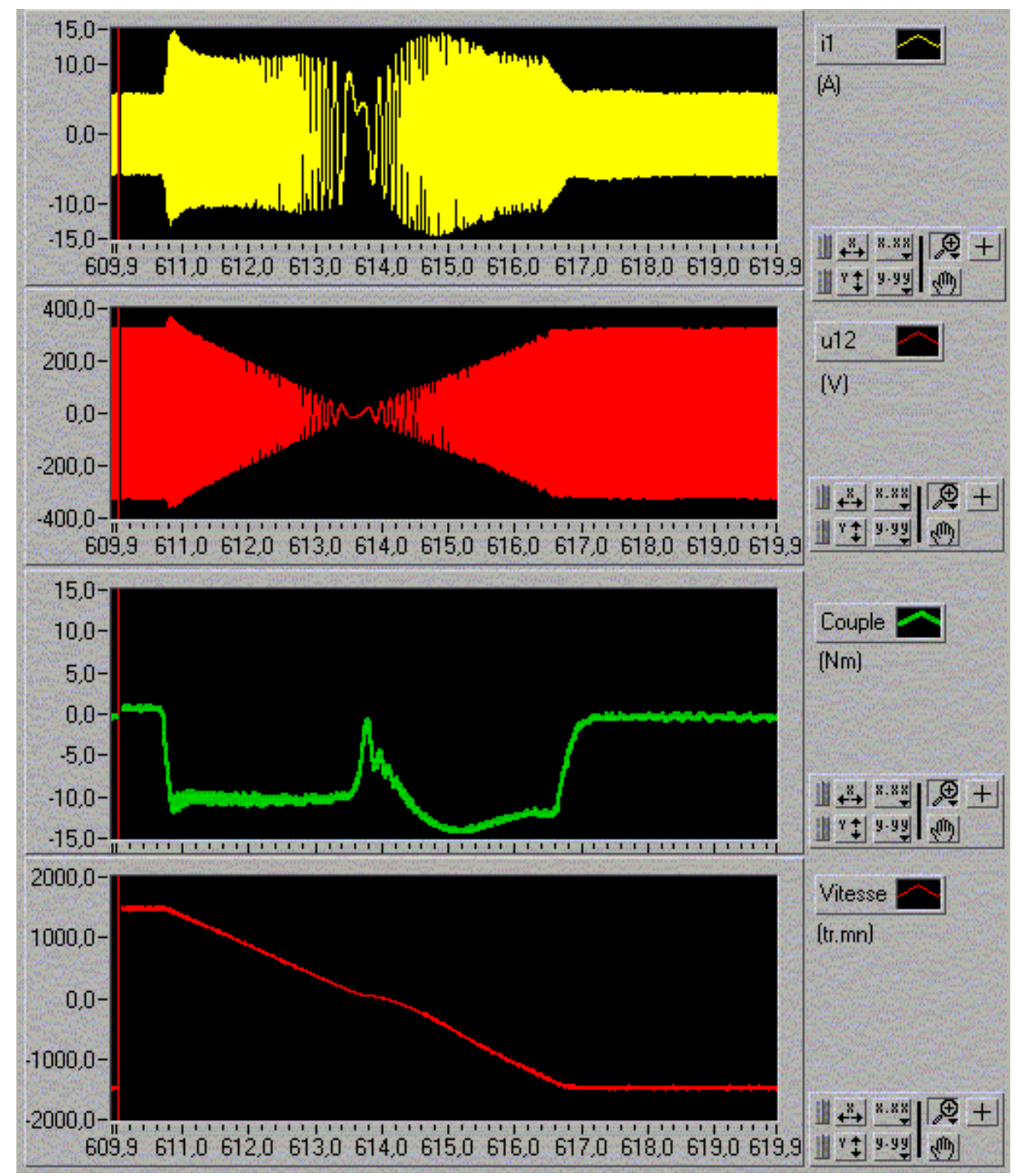

Fig. 5. Enregistrement d'un régime de fonctionnement transitoire.

\section{Conclusion}

La mise en place d'une instrumentation basée sur micro-ordinateur permet de moderniser des TPs classiques (redresseur triphasé par exemple) en les rendant plus attractifs et plus efficaces. Le temps habituellement consacré à des mesures laborieuses peut être consacré à une étude plus complète du système étudié. Dans le cas de l'étude du fonctionnement en vitesse variable d'un moteur asynchrone, des instruments virtuels spécifiques (mesures vectorielles et régimes transitoires) permettent d'aborder ce sujet de façon beaucoup plus simple et plus complète qu'avec une instrumentation classique. Le TP devient plus attractif avec une visualisation claire en temps réel qui permet une meilleure compréhension des phénomènes étudiés. 
Annexe. Diagramme LabVIEW du banc de mesure vectoriel

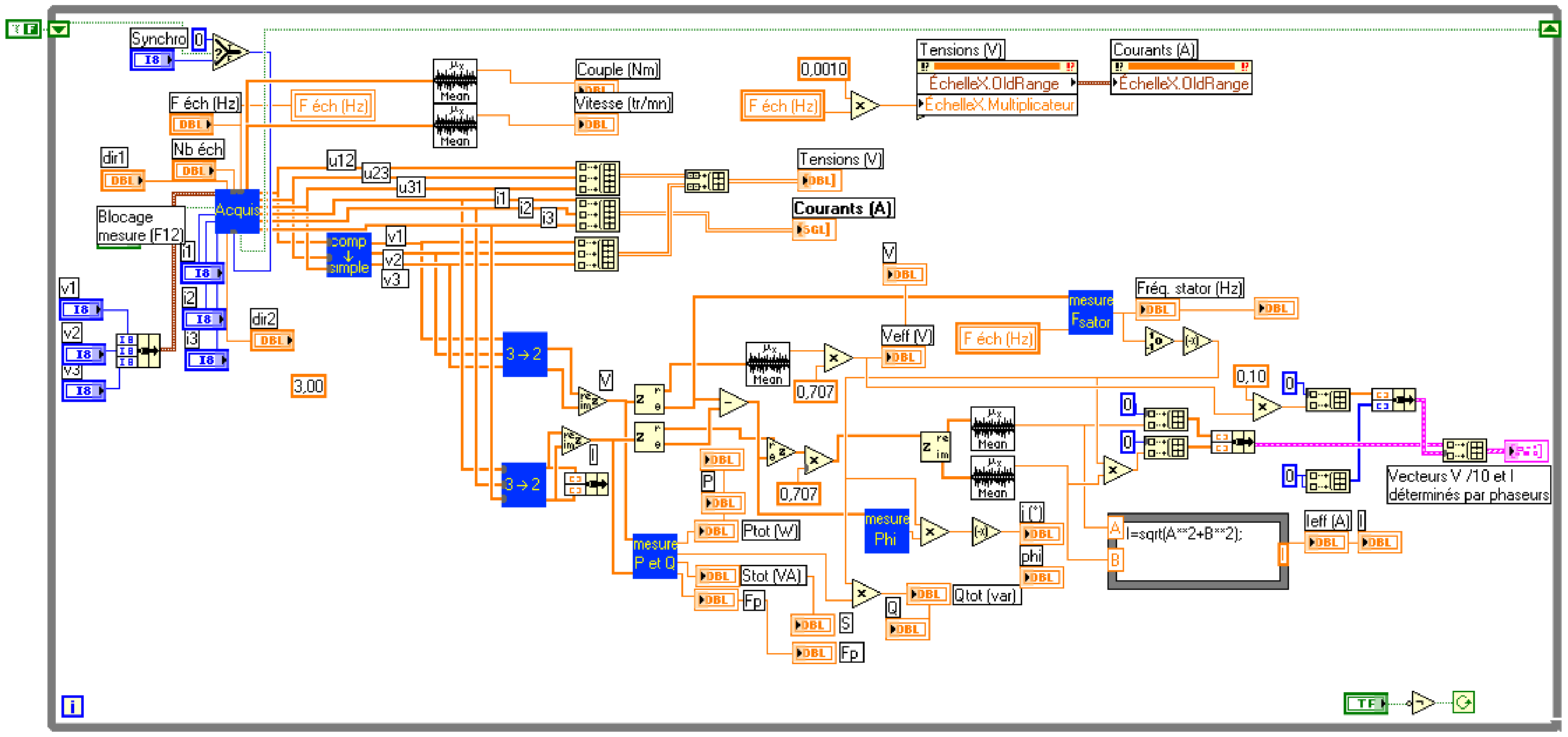

Fig. 6. Diagramme LabVIEW du banc de mesure vctoriel. 


\section{Références bibliographiques}

[1] J.-J. Huselstein, Exploitation d'un véhicule électrique en travaux pratiques d'électrotechnique, in Actes du colloque CETSISEEA'99, 4-5 novembre 1999, Montpellier, France (ISBN 2.85428.515.8) 61-64.

[2] J-J. Huselstein, Y. Patin et C. Glaize, Learning by driving on campus, in Actes de EPE/IEEE Conference $\mathrm{E}=\mathrm{TeM}$, 14-16 mars 2001, Liège, Belgique, II.77-II.85.

[3] H. Galous et al., Instrumentation d'un banc d'essai pédagogique de machine asynchrone en utilisant LabVIEW, in Actes du colloque CETSISEEA'99, 4-5 novembre 1999, Montpellier, France (ISBN 2.85428.515.8) 53-56.

[4] LabVIEW, site web de National Instruments, http://www.ni.com (consulté le 27 février 2004). 